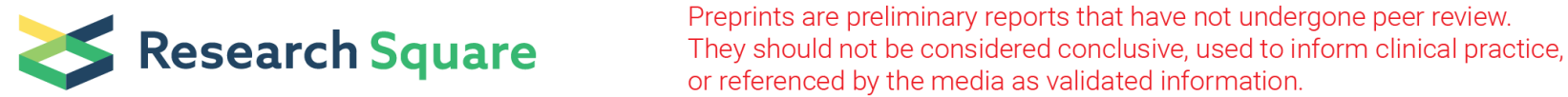

\section{Predictors Of Provider-Initiated Hiv Testing In Heterosexuals At Increased Risk Of Hiv (Het) In Puerto Rico: Data From Nhbs-Het Cycle 2016}

Maureen Marie Canario de la Torre ( $\sim$ maureen.canario@upr.edu )

Universidad de Puerto Rico Recinto de Ciencias Medicas https://orcid.org/0000-0001-5971-951X Ivony Yireth Agudelo Salas

University of Puerto Rico, Medical Sciences Campus

Sandra Miranda de León

Puerto Rico Health Department

Yadira Rolón Colón

Puerto Rico Health Department

María Pabón Martínez

Puerto Rico Health Department

Vivian Colón-López

University of Puerto Rico, Medical Sciences Campus

Research article

Keywords: HIV-Testing, Heterosexual, Provider, Puerto Rico, NHBS

Posted Date: January 20th, 2020

DOI: https://doi.org/10.21203/rs.2.21297/v1

License: (1) This work is licensed under a Creative Commons Attribution 4.0 International License.

Read Full License 


\section{Abstract}

The Centers for Disease Control and Prevention (CDC) and the World Health Organization (WHO) have established guidelines for HIV testing in healthcare settings. The aim of this study was to identify the sociodemographic, healthcare, and sexual-behavior predictors of provider-initiated HIV testing (PIHT) using data from the Puerto Rico National HIV Behavioral Surveillance (PR-NHBS) 2016 cycle directed towards heterosexuals at increased risk of HIV infection (HET). A total sample of 531 eligible participants were recruited through respondent-driven sampling (RDS). Logistic regression models assessed the associations between sociodemographic, healthcare, and sexual-behavior predictors, whilst adjusting for sex and age. The majority of the participants were women (66.1\%), with $72.7 \%$ reporting having received healthcare services in the past year. Of them, $18.7 \%$ had received an HIV-test offer from their healthcare providers. More than half of the participants (65.2\%) reported a low perceived risk of getting infected with HIV in the next 12 months. Results suggest an overall low prevalence of PIHT among HET in PR who exhibited a relatively high prevalence of low perceived risk of HIV infection. Furthermore, the assessed predictors show that individuals who engaged in high-risk sexual behaviors ( $\mathrm{AOR}=0.52 ; 95 \% \mathrm{Cl}: 0.30-$ $0.90)$ were less likely to receive HIV-test offers from their providers. This study further emphasizes the need for healthcare providers to follow recommended guidelines for HIV testing in healthcare settings as a means of establishing preventive measures to further counteract the HIV epidemic in Puerto Rico, specifically among HET.

\section{Introduction}

In $2017,38,739$ individuals were diagnosed with the human immunodeficiency virus (HIV) infection in the United States (US). Out of the total number of diagnoses, approximately $81 \%$ were among men.

Heterosexual transmission in the US is the second most common route of transmission, following that of men who have sex with men (MSM), accounting for approximately $24 \%$ of all diagnoses. ${ }^{1-3}$ As of June 30,2016 , a total of 10,243 adults and adolescents ( $\geq 13$ years) had been diagnosed with HIV infection in Puerto Rico (PR). Similar to what is seen in the US, the vast majority of the cases diagnosed on the island $(70 \%)$ are men. Heterosexual contact on the island is the most prevalent mode of transmission, accounting for $36 \%$ of the total number of cases. Women are the most affected by this route of transmission, accounting for approximately $62 \%$ of those cases $(2,271) .{ }^{4}$ Moreover, data from a population-based study documented the high prevalence of high-risk sexual behaviors among Puerto Rican men and women, highlighting the relevance of developing effective HIV prevention strategies aimed towards increasing HIV testing. ${ }^{5}$

Specifically, regarding heterosexual men and women at increased risk of HIV infection, the PR National HIV Behavioral Surveillance System (PR-NHBS) reported in their 2010 cycle that approximately $60 \%$ of these individuals (in PR) had ever been tested for HIV in their lifetime (unpublished NHBS data). This last figure reflects the need for additional HIV testing strategies for the general population. Based on the 2006 guidelines from the Centers for Disease Control and Prevention (CDC) on HIV testing in different healthcare settings, all individuals from 13 to 64 years of age should get screened for HIV infection as 
part of their routine medical examinations, and individuals at high risk should get tested annually. ${ }^{6}$ However, the number of individuals who were tested for HIV in 2010 in PR, according to the PR-NHBS, still falls short of what is expected, according to the HIV screening guidelines reported by CDC.

The World Health Organization (WHO) and the Joint United Nations Programme of HIV/AIDS (UNAIDS), recommend that healthcare providers offer opt-out HIV testing and counseling to patients. Specifically, it is suggested that HIV testing be offered to patients regardless of the presence or absence of clinical symptoms of HIV infection and the patient's motive(s) for seeking health advice. ${ }^{7}$ Such strategies ought to be implemented and practiced in all adult populations, as previous studies have shown that providerinitiated HIV testing (PIHT) has resulted in an increased number of patients who have gotten tested. 8,9

In spite of the previous recommendations, the rigorous application of this practice is not seen in most healthcare scenarios. ${ }^{10}$ Previous studies, such as that of Joore and colleagues and the systematic review by Leidel and colleagues, have identified barriers that might account for the lax implementation of PIHT. Such barriers include lack of time during routine visits, the healthcare provider's beliefs that his or her patients would feel uncomfortable discussing HIV infection, a patient's belief that his or her previous results (prior to the last 12-months) remain valid, and the provider's (or patient's) sense that such a conversation would be inappropriate because of a poor doctor-patient relationship. ${ }^{9-13}$ However, further studies have reported that such barriers might be overcome through provider initiatives such as adopting a sensitive attitude when engaging patients in discussing HIV testing and offering educational reinforcement on HIV. ${ }^{14,15}$

The burden of HIV infection in heterosexual individuals at high risk in PR and the current low HIV testing uptake highlight the need for understanding behavioral surveillance data on the predictors of PIHT. This information will be key to developing strategies to increase the uptake of HIV testing in healthcare settings. The aim of this study was to identify sociodemographic, healthcare, and sexual-behavior predictors of PIHT using data from the PR-NHBS. Insight into PIHT predictors may aid in the development of a strategy for the early identification of new HIV cases, contributing to better patient care and outcomes.

\section{Methods}

\section{Data Source}

The National HIV Behavioral Surveillance (NHBS) is a national behavioral system in the US and its jurisdictions; it aims to document high-risk behavioral practices in three different populations: men who have sex with men (MSM), persons who inject drugs (PWID), and heterosexuals at increased risk of HIV infection (HET). ${ }^{16}$ The previously mentioned populations are studied separately in rounds composed of independent cycles. The NHBS performs consecutive cross-sectional surveys in each cycle, with the aims of (1) assessing the prevalence of HIV infection, awareness, and high-risk behaviors, (2) describing HIV testing habits and the utilization of healthcare services, and (3) assessing the use of preventive 
measures. Summarized NHBS procedures are subsequently described as detailed information regarding NHBS methods can be found elsewhere. ${ }^{16}$

Data used in this study correspond to the HET cycle of the PR-NHBS's $4^{\text {th }}$ round (PR-NHBS-HET4), conducted in 2016. Recruitment for PR-NHBS-HET4 was performed through respondent-driven sampling (RDS), a technique similar to snowball sampling. ${ }^{17}$ The implementation of RDS consisted of a chain of referrals that were initiated through the establishment of non-random individuals known as "seeds." The data-collection process consisted of anonymous standardized face-to-face interviews with referred individuals who presented a valid PR-NHBS-HET4 coupon from the referral chain of RDS. Interviews were performed using a standardized questionnaire that gathered information about HIV-associated risk behaviors. Through the interview process, the participants were offered an anonymous HIV test, which they could opt out of while still completing the survey.

The participating areas were those included within the San Juan Metropolitan Statistical Area (SJ-MSA), which comprises of 41 municipalities. The estimated sample size for PR-NHBS-HET4 was 500 participants. In order to be included within the final sample, individuals must have met the following requirements at the time of the interview: They had to (1) be 18 to 60 years old, (2) have had vaginal or anal sex with a person of the opposite sex in the 12 months prior to the interview date, (3) have not previously participated in any other PR-NHBS-HET cycle, (4) live in one of the participating SJ-MSA municipalities, (5) identify as male or female (not transgender), (6) be able to complete the interview in Spanish or English, (7) have not injected drugs in the 12 months prior to the interview date, and (8) have an income falling within the Department of Health and Human Services' (HHS) poverty guidelines or have a low educational attainment no greater than a high school education. ${ }^{18,19}$ During the NHBS-HET4 cycle, a total of 599 interviews were conducted. Of that number, 68 were excluded from the study because they did not meet the inclusion criteria, leaving 531 participants in the final sample.

\section{Measurements}

Variables for sociodemographic characteristics, healthcare utilization, and HIV testing were assessed per the NHBS core questionnaire. ${ }^{20}$ Variables such as educational level, annual household income, employment, and marital status were dichotomized for data analysis. The evaluation of each individual's perception of getting infected with HIV in the next 12 months was performed using dichotomized categories. Only those participants whose answer choice was "low" were classified as positive ("yes") for perceived low risk; the answers "medium" and "high" were classified as negative ("no"). The assessment of high-risk sexual behaviors was performed through the creation of a variable which combined data from individually assessed behaviors in the NHBS-HET4 core questionnaire, similar to what was done in the study of Diepstra and colleagues. ${ }^{10}$ High-risk sexual behavior was then defined as engaging in at least one of the following: (1) any exchange sex for drugs or money, (2) having sex with more than one sexual partner, (3) having sex with a partner who "probably" or "definitely" had other sex partners, concurrently, (4) having sex with a partner who had "probably" or "definitely" injected drugs, (5) having sex with a partner who "probably" or "definitely" had had male-to-male sexual contact (only female 
respondents), and (6) having sex with a partner whose HIV status was "positive" or "indeterminate."The variable for binge drinking conformed to the operational definition from the National Institute on Alcohol Abuse and Alcoholism (NIAAA). ${ }^{21}$

\section{Data Analysis}

The database was assessed and the missing values were managed and subsequently recoded using Statistical Software Stata: Release 14. Univariate analysis assessed descriptive measurements for sociodemographic characteristics, high-risk behaviors, and the utilization of healthcare services. Bivariate analysis consisted of Pearson's chi-square and Fisher's exact tests to assess the proportional differences of the variables related to sociodemographic characteristics, HIV testing habits, high-risk behaviors, and PIHT. A multivariate logistic regression analysis assessed the interactions of statistically significant variables in the bivariate analysis. Final logistic regression models were performed whilst adjusting for sex and age. The significance level was established at 0.05 .

\section{Results}

\section{Sociodemographic Characteristics}

The total sample size consisted of 531 eligible participants. The respondents' median age was 37 years, with a standard deviation of \pm 11.94 years. The majority of the sample were women (66.1\%). A total of $434(81.7 \%)$ of the respondents reported having medical insurance at the time of the interview, and approximately $89 \%$ specified having a usual source of care from which they could seek medical advice (Table I).

\section{Utilization of Healthcare Services and HIV Testing Habits}

More than half $(72.7 \%)$ of the respondents reported having visited their healthcare providers within the last 12 months. Of these, only $18.6 \%$ were offered an HIV test by their provider. A total of 387 participants (73\%) reported having been tested for HIV infection at least one time at some point in their lifetime; of that group, only $83(27 \%)$ had gotten tested within the 12 months prior to the interview. When asked to relate the primary reasons for not getting tested for HIV in the past 12 months, the participants' most frequent answer was being afraid of having HIV (35\%), followed by no other particular reason (28\%) (data not shown). In terms of risk perception, more than half (65.2\%) of the participants reported perceiving themselves as having a low risk of getting infected with HIV in the next 12 months (Table I).

\section{Sociodemographic Characteristics, HIV Testing, High-Risk Sexual Behaviors, and PIHT}

Significant differences were observed between the participants who received an HIV-test offer by their providers and those who did not: The proportion of young individuals (18-29 years) who received an HIVtest offer from their providers was significantly higher than that of those who did not $(p<0.05)$. Contrary to this, however, the number of individuals who had engaged in high-risk sexual behaviors and who had 
received an HIV-test offer from their provider was significantly lower than the number of those who did not $(p<0.05)$. The difference in proportion of those who received the test offer vs. those who did not, tended towards statistical significance $(p=0.058)$ in women who reported engagement in high-risk sexual behaviors, (Table I). No statistical significantly difference was observed between men and women in terms of high-risk sexual behaviors (data not shown).

\section{PIHT Predictors}

Although the results were not statistically significant, multivariate logistic regression models showed that adults who exhibited the following characteristics had decreased odds of receiving an HIV-test offer from their providers: had a college education or higher (AOR $=0.72 ; 95 \% \mathrm{Cl}: 0.40-1.30)$, were single (AOR = 0.65 ; $95 \% \mathrm{Cl}$ : $0.38-1.09$ ), had an annual household income of $\$ 20,000$ or more (AOR $=0.45 ; 95 \% \mathrm{Cl}$ : $0.10-2.02)$, and had used non-injectable drugs ( $A O R=0.73 ; 95 \% \mathrm{Cl}: 0.41-1.28)$. Contrary to this, individuals who were insured $(A O R=1.21 ; 95 \% \mathrm{Cl}$ : $0.54-2.66)$, had a usual source of care where they sought medical advice $(A O R=1.96 ; 95 \% \mathrm{Cl}$ : 0.56-6.82), reported perceiving that they had a low risk of acquiring HIV (AOR = 1.20; 95\% Cl: 0.67-2.13), had used injectable drugs (AOR $=2.19 ; 95 \% \mathrm{Cl}$ : 0.54$8.85)$, or had engaged in binge drinking $(A O R=1.73 ; 95 \% \mathrm{Cl}$ : $0.89-3.34)$ had increased odds of receiving an HIV-test offer from their providers. The only significant predictor of PIHT was that of engaging in highrisk sexual behavior $(\mathrm{AOR}=0.52 ; 95 \% \mathrm{Cl}$ : $0.30-0.90)$, with the participants who reported having done so showing decreased odds of having received an HIV-test offer from their providers in the 12 months prior to the study (Table II). 


\begin{tabular}{|c|c|c|c|c|}
\hline & N (\%) & $\begin{array}{l}\text { Received HIV- } \\
\text { test offer } \\
\mathrm{n} \text { (n/total) } \\
\end{array}$ & $\begin{array}{c}\text { Did not receive HIV- } \\
\text { test offer } \\
\mathrm{n} \text { (n/total) } \\
\end{array}$ & $\begin{array}{c}p- \\
\text { value }\end{array}$ \\
\hline \multicolumn{5}{|l|}{ Sex } \\
\hline Male & 180 & $21(0.29)$ & $93(0.30)$ & \multirow[t]{2}{*}{$0.094^{\dagger}$} \\
\hline Female & $\begin{array}{l}(33.9) \\
351 \\
(66.1)\end{array}$ & $51(0.71)$ & $221(0.70)$ & \\
\hline \multicolumn{5}{|l|}{ Age } \\
\hline $18-29$ & 163 & $28(0.39)$ & $83(0.26)$ & \multirow[t]{2}{*}{$0.035^{*}$} \\
\hline $30-60$ & $\begin{array}{c}(30.7) \\
368 \\
(69.3)\end{array}$ & $44(0.61)$ & $231(0.74)$ & \\
\hline \multicolumn{5}{|l|}{ Education } \\
\hline High school or less & 374 & $53(0.74)$ & $210(0.67)$ & \multirow[t]{2}{*}{0.269} \\
\hline Some college or more & $\begin{array}{l}(70.4) \\
157 \\
(29.6)\end{array}$ & $19(0.26)$ & $104(0.33)$ & \\
\hline \multicolumn{5}{|l|}{ Employed } \\
\hline No & 339 & $41(0.57)$ & $208(0.66)$ & \multirow[t]{2}{*}{0.137} \\
\hline Full time/part time & $\begin{array}{c}(64.1) \\
190 \\
(35.9)\end{array}$ & $31(0.43)$ & $106(0.34)$ & \\
\hline \multicolumn{5}{|l|}{ Marital Status } \\
\hline Separated/divorced/widowed/never married & 325 & $35(0.49)$ & $123(0.39)$ & \multirow[t]{2}{*}{0.142} \\
\hline Married/partnered & $\begin{array}{c}(61.2) \\
206 \\
(38.8)\end{array}$ & $37(0.51)$ & $1910.61)$ & \\
\hline \multicolumn{5}{|l|}{ Currently insured } \\
\hline No & 97 & $9(0.13)$ & $44(0.14)$ & \multirow[t]{2}{*}{0.737} \\
\hline Yes & $\begin{array}{c}(18.3) \\
434 \\
(81.7)\end{array}$ & $63(0.87)$ & $270(0.86)$ & \\
\hline \multicolumn{5}{|l|}{ Lacked healthcare due to cost } \\
\hline No & 448 & $64(0.89)$ & $265(0.84)$ & \multirow[t]{2}{*}{0.332} \\
\hline Yes & $\begin{array}{c}(84.4) \\
83 \\
(15.6)\end{array}$ & $8(0.11)$ & $49(0.16)$ & \\
\hline \multicolumn{5}{|l|}{ Had a usual source of care } \\
\hline No & 59 & $3(0.04)$ & $23(0.07)$ & \multirow{2}{*}{0.440} \\
\hline Yes & $\begin{array}{l}(11.1) \\
472 \\
(88.9)\end{array}$ & $69(0.96)$ & $291(0.93)$ & \\
\hline \multicolumn{5}{|l|}{$\begin{array}{l}\text { Perceived a low risk of getting infected with HIV in } \\
\text { the next } 12 \text { months }\end{array}$} \\
\hline No & 341 & $21(0.31)$ & $109(0.35)$ & \multirow[t]{2}{*}{0.563} \\
\hline Yes & $\begin{array}{l}(65.2) \\
182 \\
(34.8)\end{array}$ & $46(0.69)$ & $202(0.65)$ & \\
\hline \multicolumn{5}{|l|}{ Engaged in high-risk sexual behavior ${ }^{b}$} \\
\hline Yes & 384 & $43(0.60)$ & $226(0.72)$ & \multirow[t]{2}{*}{$0.041^{*}$} \\
\hline ( & $\begin{array}{l}(72.3) \\
147 \\
(27.7)\end{array}$ & $29(0.40)$ & $88(0.28)$ & \\
\hline \multicolumn{5}{|l|}{ Men who engaged in high-risk sexual behavior ${ }^{b}$} \\
\hline $\begin{array}{l}\text { Yes } \\
\text { No }\end{array}$ & $\begin{array}{c}43 \\
(23.9)\end{array}$ & $\begin{array}{l}14(0.67) \\
7(0.33)\end{array}$ & $\begin{array}{l}70(0.75) \\
23(0.25)\end{array}$ & 0.419 \\
\hline
\end{tabular}


Women who engaged in high-risk sexual behavior ${ }^{\mathrm{b}}$ Yes

No
247

(70.4)

104

(29.6)

${ }^{*} \underset{* *}{p}<0.05$

** $p=0.05$

$\dagger_{0.05} \leq p<0.10$

${ }^{\mathrm{a}}$ Chi-squared test-reported $p$-value

${ }^{b}$ Defined as engaging in at least one of the following: (1) any exchange of sex for drugs or money, (2) having sex with more than one sexual partner, (3) having sex with a partner who "probably" or "definitely" had other sex partners, concurrently, (4) having sex with a partner who had "probably" or "definitely" injected drugs, (5) having sex with a partner who "probably" or "definitely" had had male-to-male sexual contact (only female respondents), and (6) having sex with a partner whose HIV status was positive or indeterminate. 


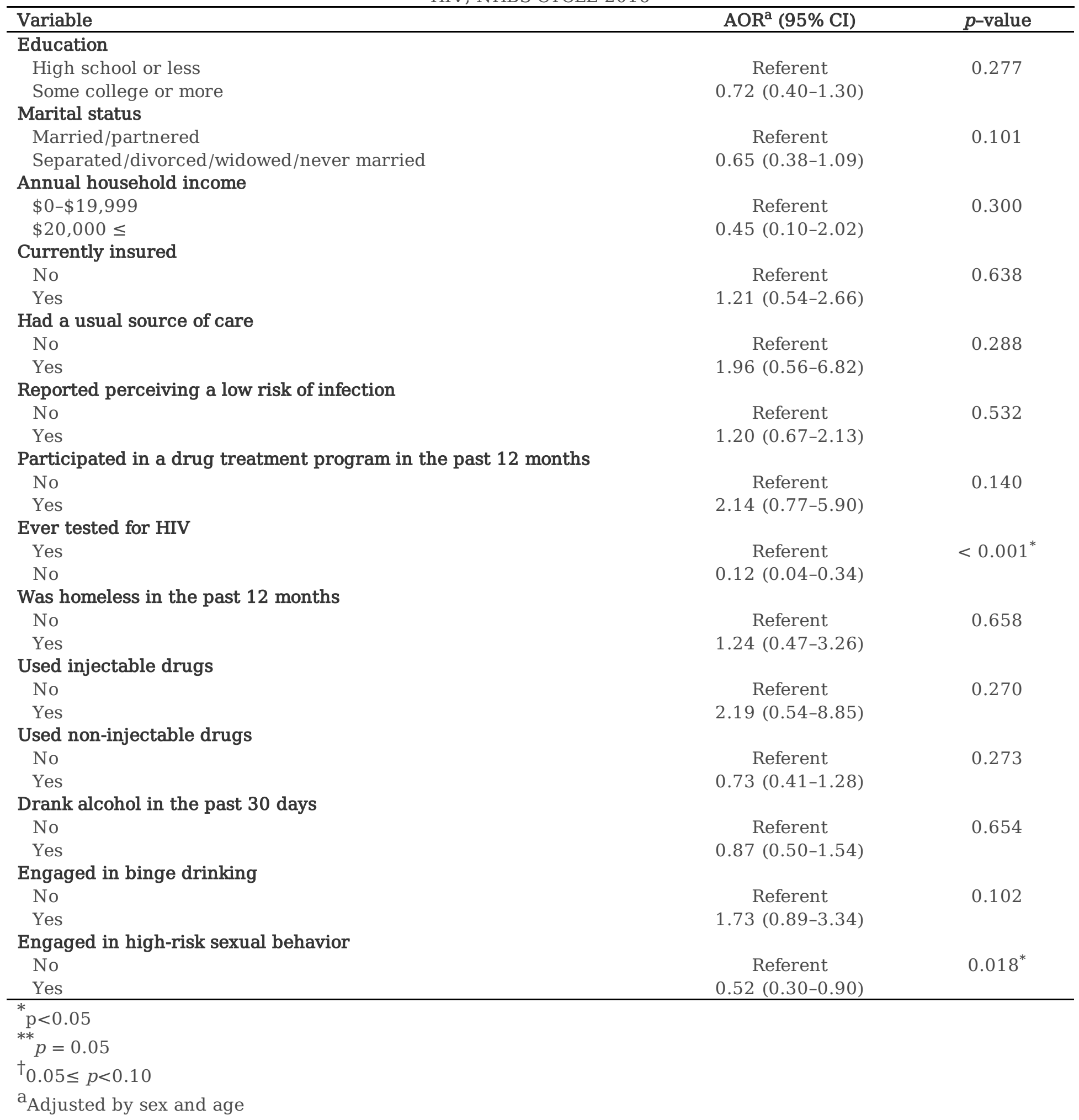

\section{Discussion}

This study aimed to determine the characteristics and behaviors that predict provider-initiated HIV-test offers. Findings show that the vast majority of the participants in this group had medical insurance coverage, had access to healthcare services, and had visited a healthcare provider in the past year. Despite their documented access to health services, a substantial proportion of these individuals had not 
received a provider-initiated HIV-test offer within the past 12 months. This finding shows a gap in the implementation of HIV testing guidelines in healthcare settings. ${ }^{6}$ Moreover, those study participants who engaged in high-risk sexual behaviors were significantly less likely to have received this type of offer. Findings also revealed that women received test offers more often than men did, in spite of there being no significant differences between the two groups in terms of reported high-risk sexual behaviors.

Similar to those of other studies that examined high-risk groups, our results showed that a high number of participants perceived themselves to be at low risk of getting infected with HIV. ${ }^{22,23}$ This perception may offer some insight into the discrepancy between the number of individuals who seek health services and those who receive an HIV-test offer, as having a low perception of risk has been previously linked to a decreased uptake of HIV testing. ${ }^{24,25}$ In addition, results for this study are congruent with those of previous studies that have established that men are less likely than women are to receive an HIV-test offer from their healthcare providers. ${ }^{26}$

Efforts to increase HIV testing on the island have recently been supported by public policy. In PR, Law Number 45 of May 2016 mandates, in accordance with CDC guidelines, that HIV testing be offered to patients as part of their routine care. Specifically, individuals from the ages of 13 to 64 years who are said to be at low-risk of infection are to be offered an HIV test as part of their routine medical testing, at least one time every five years, whilst individuals who are considered to be at high risk of infection must be offered an HIV test annually. Article 3 of this legislation specifically states that medical insurance policies, whether they be private or government issued, must include an annual HIV test in their basic health insurance coverage. ${ }^{27}$

Further strategies, in addition to that mandated by the previously implemented legislation, should be considered for rigorous implementation of PIHT and HIV awareness in order to increase HIV testing uptake among Puerto Rican HET. Efforts such as CDC-funded HIV testing events are an example of strategies proven to be effective at increasing HIV testing uptake. ${ }^{28}$ The identification of predictors of refusal to participate in PIHT can provide further insight into the barriers that limit the scope of HIV testing in healthcare settings, in terms of patients. ${ }^{29}$ Provider-targeted strategies include the implementation of PIHT isolated from patient risk assessment. Although it is recognized that patient risk assessment should be linked to HIV screening (so as to identify individuals in need of continuous screening), the findings emphasize the need for this strategy, to attempt to ensure PIHT uptake.

The limitations of the present study include the study's design. As a cross-sectional study, the impact of the evaluated variables on PIHT over time cannot be assessed. Other limitations include the use of selfreported information gathered during face-to-face interviews that could have introduced a socialdesirability bias. The studied population limits the generalization of results to other populations having different sociodemographic profiles. Contrarily, however, an advantage of the study includes the use of RDS, which has been proven to be a reliable methodology for reaching and recruiting minority populations. ${ }^{30,31}$ Despite the limitations, this behavioral surveillance system has successfully provided the necessary information for monitoring the implementation of the CDC's HIV testing guidelines and has 
made possible the better understanding of patient-driven HIV testing habits, patient healthcare access, and the risk behaviors of the members of this population, having done so with a particular focus on the Puerto Rican HET population. And lastly, compared to other studies, our results are limited due to the scope of PIHT in the literature, as most recent studies evaluated PIHT jointly with referral and counseling, indicating that patients are receptive to this joint approach. Said studies have documented the overall high levels of acceptability of PIHT when it is offered in conjunction with referrals and counseling ${ }^{8,9}$ As this study was based on PR-NHBS data collection procedures, counseling, which has been included in other studies, was not offered to the extent of providing continual care for the patient.

\section{Conclusions}

In summary, findings from this study reflect low adherence practices for HIV testing guidelines in healthcare settings among HET in PR. Increasing adherence to such guidelines is critical to ensuring the early diagnosis of unidentified HIV cases, which would lead to better patient care and outcomes as well as favor the development of more effective awareness and prevention measures. ${ }^{9,32}$ Future studies should explore the dynamics of patient and provider communication in the discussion of preventative screening. Specifically, qualitative analyses should further assess gender disparities and the underlying causes as to why women are both undergoing testing and receiving HIV-test offers more often than men are. Furthermore, a qualitative component should be added to future works in order to address or evaluate a given patient's reason or reasons for opting-out of an HIV test, identify barriers to PIHT, and collect information relevant to social factors that affect PIHT (e.g. stigma, HIV awareness, the doctor-patient relationship, etc.). As policies are enacted to support the implementation of HIV testing offered to patients as part of their routine care, implementation studies should explore the effectiveness of PIHT communication and strategies for increasing adherence to HIV testing guidelines in different healthcare settings (e.g. clinics or health centers, hospitals or emergency rooms, private doctors' offices, etc.) in PR.

\section{Declarations}

\section{Ethics approval and consent to participate}

Participation for this study required the individuals' signed consent. The NHBS-HET Cycle 2016 was submitted and approved by the University of Puerto Rico Medical Sciences Campus Institutional Review Board (IRB) under the protocol A0910115.

\section{Consent for publication}

Not applicable

\section{Availability of data and materials}

The data that support the findings of this study are available from the HIV/AIDS Surveillance Program of the Puerto Rico Health Department, but restrictions apply to the availability of these data, which were 
used under license for the current study, and so are not publicly available. Data are however available from the authors upon reasonable request and with permission of the HIV/AIDS Surveillance Program of the Puerto Rico Health Department.

\section{Competing interests}

The authors declare that they have no competing interests.

\section{Funding}

The NHBS-HET Cycle 2016 was supported by grant 6NU62PS005075-01 from the Centers for Disease Control and Prevention.

\section{Authors contributions}

As representatives from the HIV/AIDS Surveillance Program of the Puerto Rico Health Department SM, YR and MP were responsible for the conduction, field operations and data collection process of the NHBSHET Cycle 2016. Members from the Data Analysis and Publication Core (DAPuC) of the University of Puerto Rico: MC and IA performed the statistical analysis and drafting of the work; and VC oversaw the work design, interpretation and extensive review of the work. All authors read and approved the final manuscript.

\section{Acknowledgements}

Special thanks to the interviewers, nurses, and field work personnel for their diligent work during the data collection process; and to the Global Community Advisory Board of the Puerto Rico AIDS Clinical Trial Unit for their recommendations. The findings and conclusions stated in this work are those of the authors and do not necessarily represent the views of the Centers for Disease Control and Prevention.

\section{References}

1. Carney PA, O'Malley J, Buckley DI, et al. Influence of health insurance coverage on breast, cervical, and colorectal cancer screening in rural primary care settings. Cancer. 2012. doi:10.1002/cncr.27635

2. Liu Y, Sun X, Qian H-Z, et al. Qualitative Assessment of Barriers and Facilitators of Access to HIV Testing Among Men Who Have Sex with Men in China. AIDS Patient Care STDS. 2015;29(9):481-489. doi:10.1089/apc.2015.0083

3. Centers for Disease Control and Prevention. CDC FACT SHEET Today's HIV/AIDS Epidemic The Scope and Impact of HIV in the United States.

https://www.cdc.gov/nchhstp/newsroom/docs/factsheets/todaysepidemic-508.pdf. Accessed July 29, 2019. 
4. Office of Epidemiology and Research Puerto Rico Health Department. Puerto Rico (Not AIDS) Surveillance Summary.; 2016. http://www.salud.gov.pr/Estadisticas-Registros-yPublicaciones/Estadisticas VIH/Estadísticas Generales/2016/Junio 2016/Puerto Rico HIV not AIDS Surveillance Summary.pdf. Accessed July 29, 2019.

5. Patricia Ortiz A, Soto-Salgado M, Suárez E, et al. Sexual Behaviors among Adults in Puerto Rico: A Population-Based Study. doi:10.1111/j.1743-6109.2011.02329.x

6. Branson B, Handsfield HH, Lampe M, et al. Revised Recommendations for HIV Testing of Adults, Adolescents, and Pregnant Women in Health-Care Settings. https://www.cdc.gov/mmwr/preview/mmwrhtml/rr5514a1.htm. Published 2010. Accessed July 24, 2019.

7. World Health Organization. Guidance on provider-initiated HIV testing and counselling in health facilities. Heal (San Fr. 2007:1-60. https://www.who.int/hiv/topics/vct/PITC/en/. Accessed July 11, 2019.

8. Abtew S, Awoke W, Asrat A. Acceptability of provider-initiated HIV testing as an intervention for prevention of mother to child transmission of HIV and associated factors among pregnant women attending at Public Health Facilities in Assosa town, Northwest Ethiopia. BMC Res Notes. 2015;8(1):661. doi:10.1186/s13104-015-1652-4

9. Kayigamba FR, Bakker MI, Lammers J, et al. Provider-initiated hiv testing and counselling in rwanda: Acceptability among clinic attendees and workers, reasons for testing and predictors of testing. PLOS One. 2014;9(4). doi:10.1371/journal.pone.0095459

10. Diepstra KL, Cunningham T, Rhodes AG, Yerkes LE, Buyu CA. Prevalence and Predictors of ProviderInitiated HIV Test Offers Among Heterosexual Persons at Increased Risk for Acquiring HIV Infection Virginia, 2016. MMWR Morb Mortal Wkly Rep. 2018;67(25):714-717.

doi:10.15585/mmwr.mm6725a3

11. Rao S, Seth P, Walker T, et al. HIV Testing and Outcomes Among Hispanics/Latinos - United States, Puerto Rico, and U.S. Virgin Islands, 2014. MMWR Morb Mortal Wkly Rep. 2016;65(40):1099-1103. doi:10.15585/mmwr.mm6540a2

12. Leidel S, Wilson $S$, McConigley R, Boldy D, Girdler S. Health-care providers' experiences with opt-out HIV testing: A systematic review. AIDS Care - Psychol Socio-Medical Asp AIDS/HIV. 2015;27(12):14551467. doi:10.1080/09540121.2015.1058895

13. Joore IK, van Roosmalen SLC, van Bergen JEAM, van Dijk N. General practitioners' barriers and facilitators towards new provider-initiated HIV testing strategies: a qualitative study. Int J STD AIDS. 2017;28(5):459-466. doi:10.1177/0956462416652274

14. Gizaw R, Gebremdhin S. Acceptance of HIV Counseling... Acceptance of HIV Counseling and Testing among Antenatal Clinic Attendees in Southern Ethiopia. Clin Attend Hawassa City, South Ethiop Ethiop J Heal Sci. 2018;28(4):413. doi:10.4314/ejhs.v28i4.7

15. Wise JM, Ott C, Azuero A, et al. Barriers to HIV Testing: Patient and Provider Perspectives in the Deep South. AIDS Behav. 2019;23(4):1062-1072. doi:10.1007/s10461-018-02385-5 
16. Wejnert C, Broz D, Hoots B, Denning P, Paz-Bailey G. National HIV Behavioral Surveillance: Round 4 Model Surveillance Protocol.; 2015.

https://www.cdc.gov/hiv/pdf/statistics/systems/nhbs/nhbs_round4modelsurveillanceprotocol.pdf. Accessed July 26, 2019.

17. Robinson WT, Zarwell M, Gruber DA. Preferences for Home-Based HIV Testing Among Heterosexuals at Increased Risk for HIV/AIDS: New Orleans, Louisiana, 2013. J Acquir Immune Defic Syndr. 2017;75:S352-S356. doi:10.1097/QAI.0000000000001410

18. US Dept. of Health \& Human Services. 2019 Poverty Guidelines | ASPE. https://aspe.hhs.gov/2019poverty-guidelines. Accessed July 18, 2019.

19. Centers for Disease Control and Prevention. National HIV Behavioral Surveillance System Round 5: Model Surveillance Protocol. 2017;3(December).

www.cdc.gov/hiv/statistics/systems/nhbs/operations.html. Accessed July 15, 2019.

20. Centers for Disease Control and Prevention. NHBS IDU4-HET4 CAPI REFERENCE QUESTIONNAIRE (CRQ). 2017.

21. Deparment of Health and Human Services (DHHS). Drinking Levels Defined I National Institute on Alcohol Abuse and Alcoholism (NIAAA). Dietary Guidelines for Americans, 2015-2020. https://www.niaaa.nih.gov/alcohol-health/overview-alcohol-consumption/moderate-binge-drinking. Published 2015. Accessed July 18, 2019.

22. Khawcharoenporn T, Chunloy K, Apisarnthanarak A. HIV knowledge, risk perception and pre-exposure prophylaxis interest among Thai university students. Int J STD AIDS. 2015;26(14):1007-1016. doi:10.1177/0956462414564607

23. Khawcharoenporn T, Chunloy K, Apisarnthanarak A. Uptake of HIV testing and counseling, risk perception and linkage to HIV care among Thai university students. BMC Public Health. 2016;16(1):556. doi:10.1186/s12889-016-3274-8

24. Clifton S, Nardone A, Field N, et al. HIV testing, risk perception, and behaviour in the British population. AIDS. 2016;30(6):943-951. doi:10.1097/QAD.0000000000001006

25. Burns FM, Johnson AM, Nazroo J, et al. Missed opportunities for earlier HIV diagnosis within primary and secondary healthcare settings in the UK. AIDS. 2008;22(1):115-122.

doi:10.1097/QAD.0b013e3282f1d4b6

26. Dailey AF, Hoots BE, Irene Hall H, et al. Morbidity and Mortality Weekly Report Vital Signs: Human Immunodeficiency Virus Testing and Diagnosis Delays - United States. Centers Dis Control Prev. 2017;66(June). doi:10.15585/mmwr.mm6647e1

27. Lexjuris de Puerto Rico. Ley Núm. 45 de 2016 -Ley Para Ofrecer La Prueba Para El Virus de Inmunodeficiencia Humana (VIH) Como Parte de Las Pruebas de Rutina de Toda Evaluación Médica Realizada Al Menos Una Vez Cada Cinco (5) Años.; 2016.

http://www.lexjuris.com/lexlex/Leyes2016/lexI2016045.htm. Accessed September 17, 2019.

28. Hayek S, Dietz PM, Van Handel M, et al. Centers for disease control and prevention funding for HIV testing associated with higher state percentage of persons tested. J Public Heal Manag Pract. 
2015;21(6):531-537. doi:10.1097/PHH.0000000000000222

29. Facha W, Kassahun W, Workicho A. Predictors of provider- initiated HIV testing and counseling refusal by outpatient department clients in Wolaita zone, Southern Ethiopia: A case control study. BMC Public Health. 2016;16(1). doi:10.1186/s12889-016-3452-8

30. Badowski G, Somera LP, Simsiman B, et al. The efficacy of respondent-driven sampling for the health assessment of minority populations HHS Public Access. Cancer Epidemiol. 2017;50:214-220. doi:10.1016/j.canep.2017.07.006

31. Heckathorn DD. Respondent-Driven Sampling: A New Approach to the Study of Hidden Populations. Soc Probl. 2012;44(2):174-199. doi:10.2307/3096941

32. Marks G, Crepaz N, Senterfitt W, Janssen RS. Meta-Analysis of High-Risk Sexual Behavior in Persons Aware and Unaware They are Infected With HIV in the United ... Meta-Analysis of High-Risk Sexual Behavior in Persons Aware and Unaware They are Infected With HIV in the United States Implications for H. Epidemio/ Soc Sci. 2017;39:446-453. doi:10.1097/01.qai.0000151079.33935.79 